\title{
Metal flux growth of praseodymium iron carbides featuring $\mathrm{FeC}_{3}$ units
}

Ashini S. Jayasinghe and Susan E. Latturner*

Department of Chemistry and Biochemistry, Florida State University, Tallahassee FL 32306

\section{Supporting Information}

Table S1. Selected bond lengths (in $\AA$ ) in $\operatorname{Pr}_{14} \mathrm{Fe}_{6} \mathrm{C}_{13}$

\begin{tabular}{llll}
\hline $\operatorname{Pr}(1)-\mathrm{C}(2)$ & $2.6081(5)$ & $\operatorname{Pr}(3)-\operatorname{Pr}(3)$ & $3.5609(6)$ \\
$\operatorname{Pr}(1)-\mathrm{C}(3)$ & $2.906(6)$ & $\operatorname{Pr}(3)-\operatorname{Pr}(4)$ & $3.7558(4)$ \\
$\operatorname{Pr}(1)-\mathrm{C}(5)$ & $2.499(9)$ & $\operatorname{Pr}(4)-\mathrm{C}(3)$ & $2.657(6)$ \\
$\operatorname{Pr}(1)-\mathrm{Fe}(2)$ & $3.1782(8)$ & $\operatorname{Pr}(4)-\mathrm{Fe}(2)$ & $3.0371(13)$ \\
$\operatorname{Pr}(1)-\operatorname{Pr}(2)$ & $3.7224(5)$ & $\operatorname{Pr}(4)-\mathrm{Fe}(1)$ & $3.3109(10)$ \\
$\operatorname{Pr}(1)-\operatorname{Pr}(3)$ & $3.8581(5)$ & $\operatorname{Pr}(3)-\mathrm{Fe}(2)$ & $3.4748(6)$ \\
$\operatorname{Pr}(2)-\mathrm{C}(2)$ & $2.6560(6)$ & $\operatorname{Pr}(3)-\operatorname{Pr}(3)$ & $3.5609(6)$ \\
$\operatorname{Pr}(2)-\mathrm{Fe}(2)$ & $3.2176(8)$ & $\mathrm{Fe}(1)-\mathrm{C}(3)$ & $1.824(6)$ \\
$\operatorname{Pr}(2)-\operatorname{Pr}(3)$ & $3.5663(4)$ & $\mathrm{Fe}(1)-\mathrm{C}(1)$ & $1.931(5)$ \\
$\operatorname{Pr}(3)-\mathrm{C}(1)$ & $2.6092(7)$ & $\mathrm{Fe}(1)-\mathrm{Fe}(1)$ & $2.4322(17)$ \\
$\operatorname{Pr}(3)-\mathrm{C}(3)$ & $2.6746(11)$ & $\mathrm{Fe}(2)-\mathrm{C}(2)$ & $1.8163(12)$ \\
$\operatorname{Pr}(3)-\mathrm{C}(4)$ & $2.826(3)$ & $\mathrm{Fe}(2)-\mathrm{C}(3)$ & $1.822(6)$ \\
$\operatorname{Pr}(3)-\mathrm{C}(5)$ & $2.6448(11)$ & $\mathrm{C}(1)-\mathrm{C}(1)$ & $1.418(12)$ \\
$\operatorname{Pr}(3)-\mathrm{Fe}(2)$ & $3.4748(6)$ & $\mathrm{C}(4)-\mathrm{C}(5)$ & $1.378(12)$ \\
\hline
\end{tabular}


Table S2. Selected bond lengths (in $\AA$ ) in $\operatorname{Pr}_{15}\left(\mathrm{FeC}_{6}\right)_{4} \mathrm{H}$

\begin{tabular}{llll}
\hline $\operatorname{Pr}(1)-\mathrm{C}(3)$ & $2.699(6)$ & $\operatorname{Pr}(3)-\mathrm{C}(4)$ & $2.605(8)$ \\
$\operatorname{Pr}(1)-\mathrm{C}(5)$ & $2.703(11)$ & $\operatorname{Pr}(3)-\mathrm{C}(6)$ & $2.703(9)$ \\
$\operatorname{Pr}(1)-\mathrm{C}(6)$ & $2.737(11)$ & $\operatorname{Pr}(3)-\operatorname{Pr}(3)$ & $3.4155(13)$ \\
$\operatorname{Pr}(1)-\mathrm{C}(4)$ & $2.740(7)$ & $\operatorname{Pr}(4)-\mathrm{C}(1)$ & $2.703(11)$ \\
$\operatorname{Pr}(1)-\mathrm{Fe}(2)$ & $3.3994(14)$ & $\operatorname{Pr}(4)-\mathrm{C}(2)$ & $2.700(10)$ \\
$\operatorname{Pr}(1)-\mathrm{Fe}(3)$ & $3.1695(7)$ & $\operatorname{Pr}(4)-\mathrm{C}(3)$ & $3.073(6)$ \\
$\operatorname{Pr}(1)-\operatorname{Pr}(3)$ & $3.5741(7)$ & $\operatorname{Pr}(4)-\mathrm{C}(4)$ & $2.668(7)$ \\
$\operatorname{Pr}(2)-\mathrm{C}(1)$ & $2.6804(19)$ & $\operatorname{Pr}(4)-\mathrm{Fe}(1)$ & $3.1568(7)$ \\
$\operatorname{Pr}(2)-\mathrm{C}(2)$ & $2.748(3)$ & $\operatorname{Pr}(4)-\operatorname{Pr}(5)$ & $3.3537(7)$ \\
$\operatorname{Pr}(2)-\mathrm{C}(3)$ & $2.730(7)$ & $\operatorname{Pr}(5)-\mathrm{C}(2)$ & $2.660(10)$ \\
$\operatorname{Pr}(2)-\mathrm{C}(4)$ & $2.652(8)$ & $\operatorname{Pr}(5)-\mathrm{Fe}(2)$ & $2.8667(18)$ \\
$\operatorname{Pr}(2)-\mathrm{C}(5)$ & $2.728(2)$ & $\mathrm{Fe}(1)-\mathrm{C}(1)$ & $1.833(9)$ \\
$\operatorname{Pr}(2)-\mathrm{C}(6)$ & $2.919(5)$ & $\mathrm{Fe}(2)-\mathrm{C}(3)$ & $1.829(7)$ \\
$\operatorname{Pr}(2)-\mathrm{Fe}(1)$ & $3.4992(5)$ & $\mathrm{Fe}(3)-\mathrm{C}(5)$ & $1.848(10)$ \\
$\operatorname{Pr}(2)-\mathrm{Fe}(2)$ & $3.1130(6)$ & $\mathrm{C}(1)-\mathrm{C}(2)$ & $1.326(13)$ \\
$\operatorname{Pr}(2)-\mathrm{Fe}(3)$ & $3.5170(5)$ & $\mathrm{C}(3)-\mathrm{C}(4)$ & $1.330(10)$ \\
$\operatorname{Pr}(2)-\operatorname{Pr}(3)$ & $3.4497(6)$ & $\mathrm{C}(5)-\mathrm{C}(6)$ & $1.348(14)$ \\
\hline
\end{tabular}


Table S3. Selected bond lengths (in $\AA$ ) in $\operatorname{Pr}_{26} \mathrm{Fe}_{19} \mathrm{C}_{29}$

\begin{tabular}{|c|c|c|c|}
\hline $\operatorname{Pr}(1)-C(1)$ & $2.533(14)$ & $\operatorname{Pr}(5)-\mathrm{Fe}(9)$ & $3.354(2)$ \\
\hline $\operatorname{Pr}(1)-C(6)$ & $2.573(13)$ & $\operatorname{Pr}(5)-\mathrm{Fe}(1)$ & $3.418(2)$ \\
\hline $\operatorname{Pr}(1)-\mathrm{Fe}(5)$ & $2.852(3)$ & $\operatorname{Pr}(5)-\mathrm{Fe}(3)$ & $3.4265(18)$ \\
\hline $\operatorname{Pr}(1)-\mathrm{Fe}(3)$ & $2.905(3)$ & $\operatorname{Pr}(5)-\operatorname{Pr}(7)$ & 3.6611(19) \\
\hline $\operatorname{Pr}(1)-\mathrm{Fe}(2)$ & $3.242(3)$ & $\operatorname{Pr}(6)-C(4)$ & $2.605(10)$ \\
\hline $\operatorname{Pr}(1)-\mathrm{Fe}(1)$ & 3.343(3) & $\operatorname{Pr}(6)-C(2)$ & $2.632(9)$ \\
\hline $\operatorname{Pr}(1)-\operatorname{Pr}(5)$ & $3.6826(16)$ & $\operatorname{Pr}(6)-C(9)$ & $2.752(10)$ \\
\hline $\operatorname{Pr}(2)-C(9)$ & $2.531(11)$ & $\operatorname{Pr}(6)-C(1)$ & $2.804(3)$ \\
\hline $\operatorname{Pr}(2)-C(6)$ & $2.579(14)$ & $\operatorname{Pr}(6)-C(3)$ & 2.819(9) \\
\hline $\operatorname{Pr}(2)-C(5)$ & 2.584(3) & $\operatorname{Pr}(6)-\mathrm{Fe}(1)$ & $3.0967(19)$ \\
\hline $\operatorname{Pr}(2)-\mathrm{Fe}(3)$ & $2.977(2)$ & $\operatorname{Pr}(6)-\mathrm{Fe}(4)$ & $3.330(2)$ \\
\hline $\operatorname{Pr}(2)-\mathrm{Fe}(5)$ & $3.315(2)$ & $\operatorname{Pr}(6)-\mathrm{Fe}(2)$ & $3.377(2)$ \\
\hline $\operatorname{Pr}(2)-\mathrm{Fe}(4)$ & $3.390(2)$ & $\operatorname{Pr}(7)-C(7)$ & $2.544(10)$ \\
\hline $\operatorname{Pr}(2)-\mathrm{Fe}(2)$ & 3.443(3) & $\operatorname{Pr}(7)-C(2)$ & $2.605(9)$ \\
\hline $\operatorname{Pr}(2)-\operatorname{Pr}(8)$ & $3.457(2)$ & $\operatorname{Pr}(7)-C(9)$ & $2.700(10)$ \\
\hline $\operatorname{Pr}(2)-\operatorname{Pr}(3)$ & $3.6441(17)$ & $\operatorname{Pr}(7)-C(10)$ & $2.73(3)$ \\
\hline $\operatorname{Pr}(3)-C(7)$ & $2.525(10)$ & $\operatorname{Pr}(7)-C(3)$ & $2.787(10)$ \\
\hline $\operatorname{Pr}(3)-C(5)$ & $2.531(3)$ & $\operatorname{Pr}(7)-\mathrm{Fe}(6)$ & $3.257(2)$ \\
\hline $\operatorname{Pr}(3)-C(1)$ & $2.533(14)$ & $\operatorname{Pr}(7)-\mathrm{Fe}(9)$ & $3.286(2)$ \\
\hline $\operatorname{Pr}(3)-\mathrm{Fe}(5)$ & $2.913(2)$ & $\operatorname{Pr}(7)-\mathrm{Fe}(7)$ & $3.361(2)$ \\
\hline $\operatorname{Pr}(3)-\mathrm{Fe}(9)$ & 2.919(3) & $\operatorname{Pr}(7)-\mathrm{Fe}(2)$ & $3.418(2)$ \\
\hline $\operatorname{Pr}(3)-\mathrm{Fe}(4)$ & $3.223(2)$ & $\operatorname{Pr}(8)-C(9)$ & $2.529(11)$ \\
\hline $\operatorname{Pr}(3)-\mathrm{Fe}(3)$ & $3.298(2)$ & $\operatorname{Pr}(8)-C(8)$ & $2.652(14)$ \\
\hline $\operatorname{Pr}(3)-\mathrm{Fe}(1)$ & $3.319(3)$ & $\operatorname{Pr}(8)-C(10)$ & $2.78(4)$ \\
\hline $\operatorname{Pr}(3)-\operatorname{Pr}(4)$ & $3.6127(16)$ & $\operatorname{Pr}(8)-\mathrm{Fe}(8)$ & $3.200(2)$ \\
\hline $\operatorname{Pr}(4)-C(8)$ & $2.572(3)$ & $\operatorname{Pr}(8)-\mathrm{Fe}(7)$ & $3.287(3)$ \\
\hline $\operatorname{Pr}(4)-C(4)$ & $2.649(10)$ & $\operatorname{Pr}(8)-\mathrm{Fe}(6)$ & $3.302(2)$ \\
\hline $\operatorname{Pr}(4)-C(3)$ & $2.693(9)$ & $\operatorname{Pr}(8)-\operatorname{Pr}(9)$ & $3.5792(17)$ \\
\hline $\operatorname{Pr}(4)-C(7)$ & $2.790(10)$ & $\operatorname{Pr}(9)-C(4)$ & 2.627(10) \\
\hline $\operatorname{Pr}(4)-C(2)$ & $2.823(9)$ & $\operatorname{Pr}(9)-C(10)$ & $2.84(3)$ \\
\hline $\operatorname{Pr}(4)-\mathrm{Fe}(4)$ & 2.8949(18) & $\operatorname{Pr}(9)-\mathrm{Fe}(1)$ & $3.042(3)$ \\
\hline $\operatorname{Pr}(4)-\mathrm{Fe}(7)$ & $2.9574(18)$ & $\operatorname{Pr}(9)-\mathrm{Fe}(9)$ & $3.049(3)$ \\
\hline $\operatorname{Pr}(4)-\mathrm{Fe}(6)$ & $3.215(2)$ & $\operatorname{Pr}(9)-\mathrm{Fe}(8)$ & 3.139(3) \\
\hline $\operatorname{Pr}(4)-\mathrm{Fe}(8)$ & $3.3232(17)$ & $\operatorname{Pr}(9)-\mathrm{Fe}(6)$ & $3.468(2)$ \\
\hline $\operatorname{Pr}(4)-\mathrm{Fe}(1)$ & $3.353(2)$ & $\operatorname{Pr}(10)-C(8)$ & $2.686(14)$ \\
\hline $\operatorname{Pr}(4)-\operatorname{Pr}(6)$ & $3.602(2)$ & $\operatorname{Pr}(10)-C(10)$ & $2.83(4)$ \\
\hline $\operatorname{Pr}(5)-C(7)$ & $2.630(10)$ & $\operatorname{Pr}(10)-\mathrm{Fe}(8)$ & $3.003(4)$ \\
\hline $\operatorname{Pr}(5)-C(3)$ & $2.655(9)$ & $\operatorname{Pr}(10)-\mathrm{Fe}(7)$ & $3.407(3)$ \\
\hline $\operatorname{Pr}(5)-C(6)$ & $2.686(3)$ & $\mathrm{Fe}(1)-\mathrm{C}(1)$ & $1.801(14)$ \\
\hline $\operatorname{Pr}(5)-C(4)$ & $2.723(10)$ & $\mathrm{Fe}(2)-\mathrm{C}(6)$ & $1.820(14)$ \\
\hline $\operatorname{Pr}(5)-C(2)$ & $2.850(9)$ & $\mathrm{Fe}(2)-\mathrm{C}(2)$ & $1.984(10)$ \\
\hline $\operatorname{Pr}(5)-\mathrm{Fe}(6)$ & $2.9363(18)$ & $\mathrm{Fe}(2)-\mathrm{Fe}(7)$ & $2.352(3)$ \\
\hline $\operatorname{Pr}(5)-\mathrm{Fe}(2)$ & 3.0316(18) & $\mathrm{Fe}(4)-\mathrm{Fe}(6)$ & $2.396(2)$ \\
\hline $\operatorname{Pr}(5)-\mathrm{Fe}(4)$ & $3.201(2)$ & $\mathrm{C}(2)-\mathrm{C}(3)$ & $1.385(13)$ \\
\hline
\end{tabular}



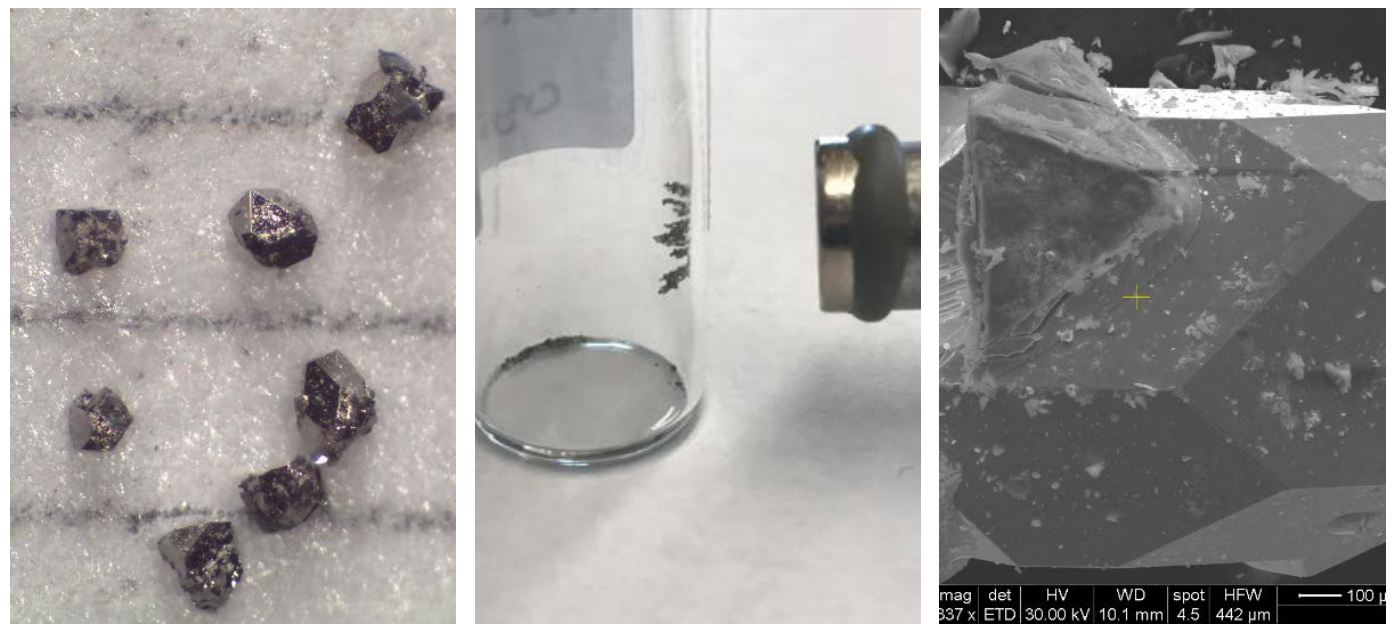

Figure SI1. $\operatorname{Pr}_{2} \mathrm{Fe}_{17} \mathrm{C}_{\mathrm{x}}$ forms as a byproduct in the presence of $\operatorname{Pr}_{15}\left(\mathrm{FeC}_{6}\right)_{4} \mathrm{H}$; this byproduct is ferromagnetic at room temperature.

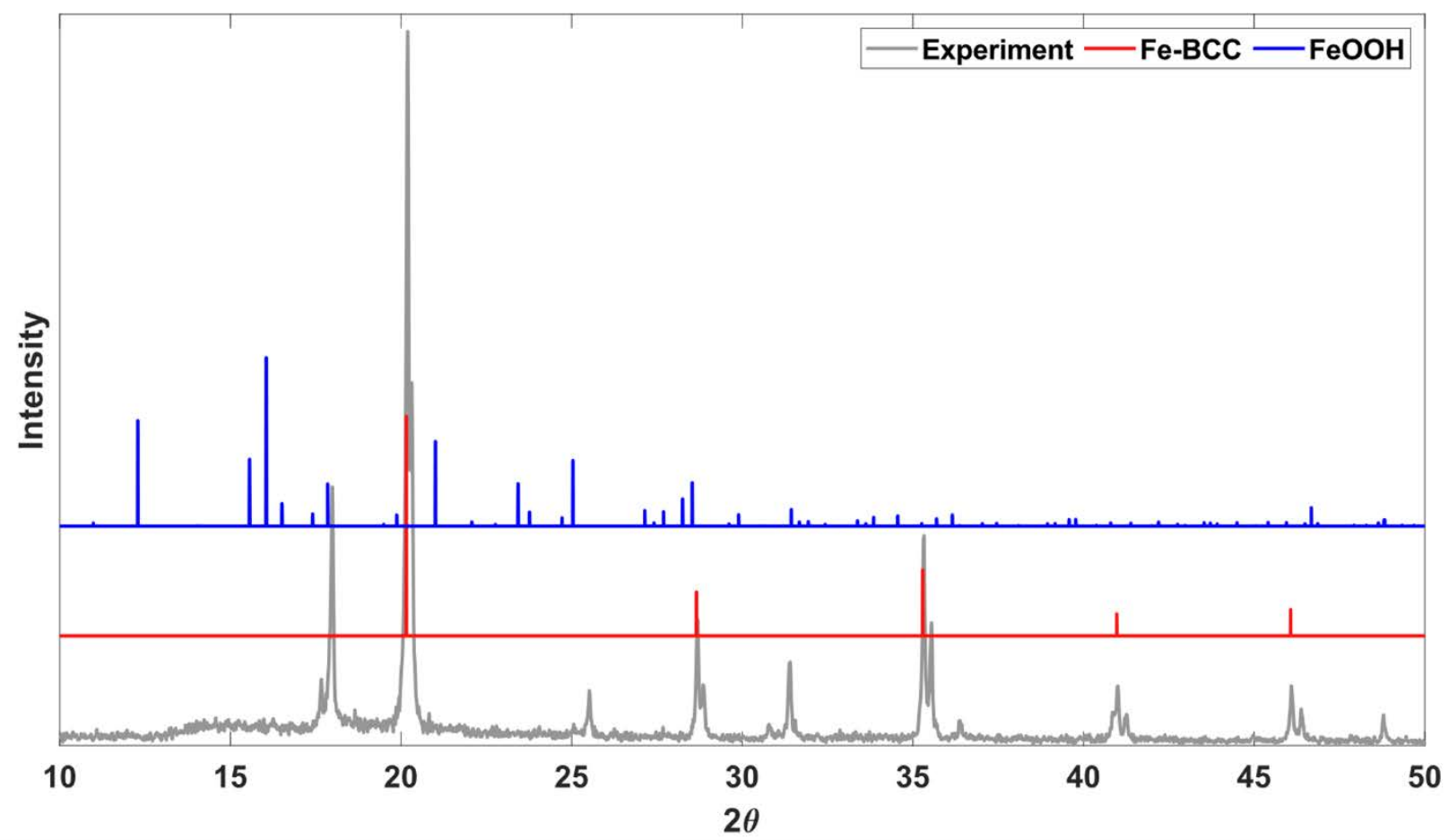

Figure SI2. Powder X-ray diffraction data of Fe powder used in the experiments compared with calculated Fe and $\mathrm{FeOOH}$ powder patterns. 